\title{
NUMERIC RATING SCALE ANALYSIS OF TRIGEMINAL NEURALGIA PATIENTS BEFORE AND AFTER MICROVASCULAR DECOMPRESSION
}

\author{
Elena Ghentilis Fitri Amelia ${ }^{1}$, Agus Turchan ${ }^{2}$, Nancy Margarita Rehatta ${ }^{3}$, Hanik Badriyah Hidayati ${ }^{4}$ \\ Correspondence: hanikhidayati@yahoo.com \\ ${ }^{I}$ Medicine Study Program, Faculty of Medicine, Airlangga University, Surabaya, Indonesia \\ ${ }^{2}$ Department of Neurosurgery, Faculty of Medicine, Airlangga University, Surabaya, Indonesia \\ ${ }^{3}$ Department of Anesthesiology and Reanimation, Faculty of Medicine, Airlangga University, Surabaya, Indonesia \\ ${ }^{4}$ Department of Neurology, Faculty of Medicine, Airlangga University, Surabaya, Indonesia
}

Article History:

Received: December 28, 2019

Accepted: September 29, 2020

Published: January 1, 2020

\section{Cite this as:}

Amelia EGF, Turchan A, Rehatto

$N M$, Hidayati HB. Numeric

rating scale analysis of

trigeminal neuralgia patients

before and after microvascular

decompression. Malang

Neurology Journal; 2021.7:17-

19.

http://dx.doi.org/10.21776/ub.mnj 2021.007.01.4

\section{ABSTRACT}

Background: Trigeminal Neuralgia is a common condition of facial pain and its significantly affect patients' daily life. Microvascular decompression is one of the interventional pain management for trigeminal neuralgia. There is still a little data obtained on evaluation of facial pain after microvascular decompression in Indonesia.

Objective: This research aimed to evaluate facial pain after microvascular decompression of trigeminal neuralgia patients in Dr. Soetomo General Hospital, PHC Hospital, and Bangil General Hospital, Indonesia.

Methods: The research design was a pretest-posttest with total sampling. Data were obtained from medical records from January 2018 until June 2019. Researches used Numeric Rating Scale (NRS) as pain measurement. The data obtained were analyzed by descriptive statistical test, normality test, and paired t-test.

Results: Trigeminal Neuralgia patients that has been treated with microvascular decompression have an average facial reduction from $7.33 \pm 2.29$ to $1.89 \pm 3.41$ with $\mathrm{p}=0.001$. This result showed that the microvascular decompression has significantly reduce facial pain in patients with trigeminal neuralgia. Conclusion: Microvascular decompression significantly reduce the facial pain of trigeminal neuralgia patients.

Keywords: Numeric rating scale, trigeminal neuralgia, microvascular decompression

\section{Introduction}

Trigeminal neuralgia (TN) is a condition characterized by unilateral paroxysmal of severe lancinating-like pain in the distribution of the fifth cranial nerve. ${ }^{1}$

The annual incidence of $\mathrm{TN}$ is approximately 27 per 100,000 people per year, with higher frequency in patient between the ages of 37-67..$^{2,3}$ The prevalence of TN was found higher in women, vary between $0.03 \%$ to $0.3 \% .^{3}$

The neuropathic pain felt by the TN patients result in significant impairment of the quality of life such as difficulty to wash their face, to shave, and to maintain oral hygiene. ${ }^{4}$ Patients tend to live in fear toward the next attack and the severity of the pain will be felt. As a consequence of it, depression and anxiety are highly found in TN patients. $^{5}$

There is an abundant amount of therapeutic options for trigeminal neuralgia, such as pharmacological therapy and non-pharmacological therapy. In the management of EAN explained that the first line of trigeminal neuralgia therapy is the administration of carbamazepine and oxcarbazepine for the long-term treatment. ${ }^{6}$ Surgical therapy is required if an unbearable pain is still perceived after the following pharmacological treatment procedure or if the patients experience side effects from the drugs consumed. By directly intervening in the location of pain, it is expected to be able to cure the pain permanently. Surgical options offered are rhizotomy, peripheral neurectomy, microvascular decompression, and others. Rhizotomy is a surgical technique which an injury is made in the trigeminal ganglion through the foramen ovale. Unfortunately, his surgical procedure conveys high possibility of recurrence. ${ }^{7}$ Other actions that can be done are cutting the trigeminal nerve branches or peripheral neurectomy. Peripheral neurectomy is linked to provide only short to medium-term pain relief. ${ }^{8}$ Microvascular Decompression (MVD) is the gold standard therapy for trigeminal neuralgia that manages to provide the highest success rate. ${ }^{7}$ According to Technical Note from Janneta MVD is done by providing a barrier between blood vessels that run side by side with the trigeminal nerve. It aims to reduce mechanical stimulation in the form of pulses of blood vessels so that the pain felt disappears. ${ }^{9}$

Evaluation of pain is important to evaluate the success rate of therapy. This study evaluates facial pain reduction after TN patients underwent microvascular decompression. 


\section{Methods}

The design of this research was pretest-posttest. Data were collected from 9 medical record of patient since January 2017 until June 2019. Sample were collected by total sampling method. Population includes all trigeminal neuralgia patients in Dr. Soetomo General Hospital, PHC Hospital, and Bangil General Hospital. The patients who meet inclusion criteria were included in the research. Inclusion criteria: age more than 25, diagnosed with trigeminal neuralgia, and underwent microvascular decompression surgery. Exclusion criteria: patients who were not willing to be the subject.

The data is collected by observing the medical record and interviewing patients of trigeminal neuralgia. Demographic and clinical data (age, sex, pain assessment) were registered. Pain was assessed with an eleven-item NRS (010), where patient rate their pre-surgery and post-surgery pain intensity. The pain reduction then was analyzed using paired t-test. Data analysis used IBM SPSS Statistics 20.

The scientific ethical committee of Dr. Soetomo General Hospital has approved the research (ethic clearance number: 0711/KEPK/X/2018) and safety monitoring is done by maintaining patients' data confidentiality.

\section{Results}

\section{Demographic data}

A total of 9 patient were included in the study. There were $6(66.7 \%)$ female patients and $3(33.3 \%)$ male patients. The most frequent patients are female patients in the age range of $46-55$ years which is 2 patients $(22.2 \%)$. The demographic data of patients are shown in Table 1.

Table 1. Demographic table of patients based on gender and age

\begin{tabular}{|c|c|c|c|}
\hline \multirow{2}{*}{$\begin{array}{c}\text { Age } \\
\text { (year) }\end{array}$} & \multicolumn{2}{|c|}{ Gender } & \multirow{2}{*}{$\begin{array}{c}\text { Total } \\
\text { Percentage } \\
(\%)\end{array}$} \\
\hline & Number of Patients (n) & $\frac{\text { itients (n) }}{\text { Female }}$ & \\
\hline $17-25$ & $0(0.0 \%)$ & $1(11.1 \%)$ & 11.1 \\
\hline $26-35$ & $0(0.0 \%)$ & $1(11.1 \%)$ & 11.1 \\
\hline $36-45$ & $1(11.1 \%)$ & $1(11.1 \%)$ & 22.2 \\
\hline $46-55$ & $0(0.0 \%)$ & $2(22.2 \%)$ & 22.2 \\
\hline $56-65$ & $1(11.1 \%)$ & $1(11.1 \%)$ & 22.2 \\
\hline$>65$ & $1(11.1 \%)$ & $0(0.0 \%)$ & 1.11 \\
\hline otal (n) & $3(33.3 \%)$ & $6(66.7 \%)$ & 100 \\
\hline
\end{tabular}

Comparison of NRS before and After Microvascular Decompression

The mean \pm standard deviation (SD) NRS preoperative was $7.33 \pm 2.29$. After treated with microvascular decompression, each patient demonstrated significant improvement. The mean \pm SD change (improvement) score for NRS was $5.44 \pm 3.13$. No patients experienced recurrence and complication. A significant difference in pain measurement was observed between preoperative and postoperative $(P=0.001)$. The comparison data between NRS before and after microvascular decompression are shown in Table 2.

Table 2. Comparison of NRS Before and After Microvascular Decompression

\begin{tabular}{lllll}
\hline & Preoperative & Postoperative & Difference & $\boldsymbol{p}$ value \\
\hline NRS & $7.33 \pm 2.29$ & $1.89 \pm 3.41$ & $5.44 \pm 3.13$ & 0.001 \\
\hline
\end{tabular}

\section{Discussion}

Numeric Rating Scale is one of the pain measurements that can be used to determine the pain intensity felt by $\mathrm{TN}$ patients. TN occurred due to vascular compression in trigeminal nerve root, causing morphological changes. ${ }^{10}$ One form of intervention that can be done to reduce the pain is surgery. Microvascular decompression is a surgery which focus on eliminating vascular compression and resulting in pain reduction that can be measured with NRS.

The findings of the current study highlight the significant pain reduction felt by $\mathrm{TN}$ patients. The detailed analyses indicate that there is an average of $5.44 \pm 3.13$ pain reduction reported by all patient who underwent microvascular decompression. A 2018 study conducted by Jiao et al. displayed $88.9 \%$ cases have total pain reduction after 2 years evaluation after microvascular decompression. ${ }^{11}$ Unfortunately, in our study there are difference in time range of pain measurement after microvascular decompression that may become a confounding variable in the pain scale of each patient.

In this study, microvascular decompression showed a good outcome in treating $\mathrm{TN}$, no matter how severe the facial pain and intractable with other therapy before microvascular decompression. A technique introduced by Janneta in 1967, is known as the most useful intervention for TN cases. ${ }^{9}$ Microvascular decompression can totally reduce the pain felt by TN patients because its target is the etiology of TN itself. The concept that underlies Janneta developing the microvascular decompression technique is the concept of neurovascular compression theory from Dandy and Gardner, which said that normal nerves can cause cranial nerve disorder due to compression of blood vessels in related nerve roots. ${ }^{9}$ The administration of a Teflon barrier between nerves and blood vessels can reduce mechanical stimulation from the pulses of related blood vessels which results in disappearance of the facial pain felt by patients. ${ }^{12}$

Arteries compression have been associated with good outcomes for TN patients. ${ }^{13}$ Arteries compression will give better results in compared to venous compression because the compression is not so severe and maximum decompression can be performed. ${ }^{13}$ In general, the arteries that cause the most trigeminal neuralgia are the anterior cerebelli arteries. ${ }^{14}$ In line with this statement, a 2018 study showed that most trigeminal neuralgia is caused by the superior cerebellar artery, followed by the superior petrous vein, the basilar artery, and the arachnoid membrane. ${ }^{11}$ In our study, no good records carried out so further research is needed to determine various factors causing good outcomes from microvascular decompression.

Almost all studies say that there is a $0.1 \%$ chance of mortality and complications that may arise after surgery, this surgery must be performed by surgeons who are experienced in their field. ${ }^{15}$ In the previous study, microvascular decompression may showed complications such as cranial nerve deficits, postoperative CSF leak, cerebellar injury, and sensory disturbances. ${ }^{16}$ Another study also reported that the follow-up examination found hearing loss after patients treated. ${ }^{17,18}$ These results are still not completely reliable because it is still unclear whether hearing loss has been seen from before giving the 
intervention. ${ }^{18}$ In contrast to the results of previous studies, the results of research conducted at Dr. Soetomo General Hospital, PHC Hospital, and Bangil General Hospital showed no complications experienced by patients after underwent microvascular decompression.

\section{Conclusion}

In conclusion, microvascular decompression provides significant pain reduction measured in Numeric Rating Scale (NRS) for trigeminal neuralgia patients treated by Dr. Soetomo General Hospital, PHC Hospital, and Bangil General Hospital neurosurgeon team.

\section{Acknowledgement}

The researchers would like to thank to Airlangga University, Dr. Soetomo General Hospital, PHC Hospital, and Bangil General Hospital for facilitating this research.

\section{References}

1. Cheng J, Long J, Hui X, Lei D, Zhang H. Effects of microvascular decompression on depression and anxiety in trigeminal neuralgia: A prospective cohort study focused on risk factors and prognosis. Clin Neurol Neurosurg. 2017.

DOI: 10.1016/j.clineuro.2017.08.011

2. Hannan C, Shoakazemi A, Quigley G. Microvascular decompression for trigeminal neuralgia: A regional unit's experience. Ulster Med J; 2018.

3. De Toledo IP, Conti Réus J, Fernandes M, et al. Prevalence of trigeminal neuralgia: A systematic review. J Am Dent Assoc. 2016.

DOI :10.1016/j.adaj.2016.02.014

4. Smith JG, Elias L-A, Yilmaz Z, et al. The psychosocial and affective burden of posttraumatic stress neuropathy following injuries to the trigeminal nerve. J Orofac Pain; 2013.

DOI: $10.11607 /$ jop.1056

5. Zakrzewska JM, Wu J, Mon-Williams M, Phillips N, Pavitt SH. Evaluating the impact of trigeminal neuralgia. Pain; 2017.

DOI: 10.1097/j.pain.0000000000000853

6. Bendtsen L, Zakrzewska JM, Abbott J, et al. EAN guideline on trigeminal neuralgia. Eur J Neurol; 2019. DOI :10.1111/ene. 13950

7. Bick SKB, Eskandar EN. Surgical treatment of trigeminal neuralgia. Neurosurg Clin N Am; 2017. DOI:10.1016/j.nec.2017.02.009

8. Yuvaraj V, Krishnan B, Therese BA, Balaji TS.
Efficacy of neurectomy of peripheral branches of the trigeminal nerve in trigeminal neuralgia: A critical review of the literature. J Maxillofac Oral Surg; 2019. DOI :10.1007/s12663-018-1108-1

9. Kaufmann AM, Price A V. A history of the Jannetta procedure. J Neurosurg; 2019. DOI : $10.3171 / 2018.10 . j n s 181983$

10. Cruccu G, Finnerup NB, Jensen TS, et al. Trigeminal neuralgia: New classification and diagnostic grading for practice and research. Neurology; 2016.

DOI: 10.1212/WNL.0000000000002840

11. Jiao Y, Yan Z, Che S, et al. Improved Microvascular Decompression in Treating Trigeminal Neuralgia: Application of Nest-Shaped Teflon Fibers. World Neurosurg; 2018. DOI: 10.1016/j.wneu.2017.09.138

12. Wang DD, Raygor KP, Cage TA, et al. Prospective comparison of long-term pain relief rates after frsttime microvascular decompression and stereotactic radiosurgery for trigeminal neuralgia. J Neurosurg; 2018. DOI: $10.3171 / 2016.9 . J N S 16149$

13. Jafree DJ, Zakrzewska JM. Long-term pain relief at five years after medical, repeat surgical procedures or no management for recurrence of trigeminal neuralgia after microvascular decompression: Analysis of a historical cohort. Br J Neurosurg; 2019. DOI: 10.1080/02688697.2018.1538484

14. Gajski D, Dennis AR, Arnautovic KI. Microsurgical decompression of trigeminal neuralgia caused by simultaneous double arterial (SCA and AICA) and petrosal vein complex compression. J Neurol Surgery, Part B Skull Base; 2018. DOI: 10.1055/s-00381669968

15. Montano N, Conforti G, Di Bonaventura R, Meglio M, Fernandez E, Papacci F. Advances in diagnosis and treatment of trigeminal neuralgia. Ther Clin Risk Manag; 2015. DOI: 10.2147/TCRM.S37592

16. Tomasello F, Esposito F, Abbritti R V., et al. Microvascular decompression for trigeminal neuralgia: Technical refinement for complication avoidance. World Neurosurg; 2016.

DOI: 10.1016/j.wneu.2016.06.097

17. Bendtsen L, Zakrzewska JM, Abbott J, et al. European academy of neurology guideline on trigeminal neuralgia. Eur J Neurol; 2019.

DOI: $10.1111 /$ ene. 13950

18. Sandell T, Eide PK. The effect of microvascular decompression in patients with multiple sclerosis and trigeminal neuralgia. Neurosurgery; 2010.

DOI: 10.1227/01.NEU.0000375491.81803.5D 